\title{
Crescimento e fixação biológica do nitrogênio em soja cultivada com doses de lodo de esgoto compostado
}

\author{
Growth and nitrogen fixation in soybean treated with doses of \\ composted sewage sludge
}

\author{
Thomaz Figueiredo Lobo ${ }^{1 *}$; Helio Grassi Filho²; Elke Jurandy Bran Nogueira \\ Cardoso $^{3}$; Leandro de Souza Almeida ${ }^{4}$; Norio Nomiyama Junior ${ }^{4}$
}

\begin{abstract}
Resumo
A utilização de lodo de esgoto é uma prática altamente promissora para o desenvolvimento de sistemas agrícolas sustentáveis. O objetivo deste trabalho foi avaliar doses de lodo de esgoto compostado, com e sem a inoculação do rizóbio, no teor de $\mathrm{N}$ foliar, número de nódulos, massa seca de nódulos e da planta no florescimento. $\mathrm{O}$ experimento foi conduzido em casa de vegetação do Departamento de Ciência do Solo e Recursos Naturais da Faculdade de Ciências Agronômicas de Botucatu, utilizando como substrato vasos de 30 litros de um Latossolo Vermelho Amarelo com textura arenosa Foi adotado o delineamento experimental em blocos casualizados constituído por 10 tratamentos, sendo cinco doses de lodo de esgoto compostado $\left(0,10,20,30,40 \mathrm{t} \mathrm{ha}^{-1}\right)$ com ou sem a inoculação do Bradyrizobium Japonic, com 3 repetições. Houve acréscimo no número e massa seca dos nódulos e na massa seca da parte aérea da soja com o aumento da dose de lodo até $20 \mathrm{t} \mathrm{ha}^{-1} \mathrm{e}$, posterior a esta dose ocorreram decréscimos destes parâmetros. Na dose de 10 t ha $^{-1}$ de lodo compostado as sementes inoculadas apresentaram superior para os teores foliares de $\mathrm{N}$ e número de nódulos comparados com as sementes não inoculadas. Na dose de $30 \mathrm{t} \mathrm{ha}^{-1}$ as plantas provenientes de sementes inoculadas foram superiores em relação a não inoculadas em todos os parâmetros analisados.
\end{abstract}

Palavras-chave: Microbiologia do solo, biossólido, rizóbio, sustentabilidade

\begin{abstract}
The use of sewage sludge is a highly promising practice for the development of sustainable agricultural systems. The objective of this study was to evaluate doses of sewage sludge composted with and without Rhizobium inoculation in leaf $\mathrm{N}$ content, nodule number, nodule dry weight and plant during flowering. The experiment was conducted in the greenhouse of the Department of Soil Science and Natural Resources College of Agricultural Sciences of Botucatu, using as substrate used in vessels of 30 liters a Red Yelow Latosol sandy texture with experimental design adopted was randomized blocks constituted for 10 treatments and five doses of composted sewage sludge $\left(0,10,20,30,40 \mathrm{tha}^{-1}\right)$ with or without inoculation Bradyrhizobium japonic with three replications. There was an increase in the number and dry weight of nodules and shoot dry mass of soybeans due to the increase of the dose of
\end{abstract}

\footnotetext{
${ }^{1}$ Eng $^{\circ}$ Agr $^{\circ}$, pós-doutorando do curso de irrigação. Universidade Estadual Paulista "Julio de Mesquita Filho", UNESP, Botucatu, SP. E-mail: thomaz.lobo@superig.com.br

${ }^{2}$ Eng $^{\mathrm{o}}$ Agr $^{\mathrm{o}}$, Dr. UNESP, Dept ${ }^{\mathrm{o}}$ de Recursos Naturais e Ciência do Solo, Fazenda Experimental Lageado, Botucatu, SP. E-mail: heliograsi@fca.unesp.br

${ }^{3}$ Eng $^{\mathrm{a}} \mathrm{Agr}^{\mathrm{a}}$, Dr ${ }^{\mathrm{a}}$. Universidade São Paulo, ESALQ, Dept ${ }^{\circ}$ de Solos e Nutrição de Plantas. Prof ${ }^{\mathrm{a}}$ Titular, Microbiologia e Bioquímica do Solo, Piracicaba, SP. E-mail: ejbncard@esalq.usp.br

${ }^{4}$ Discente(s) de graduação, UNESP, Dept ${ }^{\circ}$ de Recursos Naturais e Ciência do Solo, Fazenda Experimental Lageado, Botucatu, SP. E-mail: ldsalmeida@fca.unesp.br; nnjunior@fca.unesp.br; akfrancisco@fca.unesp.br

* Autor para correspondência
} 
sludge up to a dose of $20 \mathrm{t} \mathrm{ha}^{-1}$ and after this dose there was a decrease of these parameters. At a dose of $10 \mathrm{t} \mathrm{ha}^{-1}$ sludge compost inoculated seeds showed higher for foliar concentrations of $\mathrm{N}$ and number of nodules compared with uninoculated seeds. At a dose of $30 \mathrm{t} \mathrm{ha}^{-1}$ inoculated seeds were higher compared to uninoculated in all parameters.

Key words: Microbiology of soil, biosolids, rhizobium, sustainability

\section{Introdução}

A disposição final do lodo de esgoto vem se caracterizando como um dos problemas ambientais urbanos mais relevantes da atualidade e que cresce diariamente tanto nos países desenvolvidos quanto naqueles em desenvolvimento, como reflexo da ampliação das redes de coleta e incremento dos níveis de tratamento do esgoto (BETTIOL; CAMARGO, 2006).

O uso agrícola do lodo de esgoto como adubo orgânico é considerado hoje como alternativo mais promissor de disposição final deste resíduo (BERTON; NOGUEIRA, 2010). O seu efeito pode ser potencializado, aliando-se a utilização agrícola e a recuperação de áreas degradadas.

A utilização de lodo de esgoto é uma prática altamente promissora para o desenvolvimento de sistemas agrícolas sustentáveis. No entanto, normas rígidas devem ser seguidas para minimizar o impacto desta prática no ambiente, como a norma técnica P 4.240 (CETESB, 1999).

Na dose de $30 \mathrm{t} \mathrm{ha}^{-1}$ de lodo, base úmida, há viabilidade econômica para dois cultivos de soja, com retorno de $\mathrm{R} \$ 0,15$ para cada $\mathrm{R} \$ 1,00$ investido no lodo como fertilizante. Na cultura do milho o retorno pode chegar a $\mathrm{R} \$ 0,90$ (EMBRAPA, 2007).

$\mathrm{O}$ lodo de esgoto pode liberar para a solução do solo grande quantidade de $\mathrm{N}$ mineral nos primeiros dias após a aplicação, se houver condições propícias à mineralização da matéria orgânica (BOEIRA, 2004). Entretanto, após a compostagem com outros resíduos agrícolas, a relação $\mathrm{C} / \mathrm{N}$ atinge valores mais aceitáveis e considerados adequados para uma aplicação no solo sem causar problemas ambientais por causa do excesso de $\mathrm{N}$.
Cordeiro (1977) relata que, para produzir 2.000 $\mathrm{kg} \mathrm{ha}^{-1}$ de grãos de soja, são necessários cerca de 170 $\mathrm{kg}$ de N, dos quais $110 \mathrm{~kg}$ são exportados pelos grãos. Mas, diversos trabalhos citados por Campo e Sfredo (1981) mostraram que o N é inibidor da nodulação e, conseqüentemente, da fixação simbiótica de $\mathrm{N}$. Os trabalhos conduzidos por Campo, Palhano e Lantmann (1981), em solos do Paraná, utilizando doses de $\mathrm{N}$ no sulco de semeadura, também não revelaram acréscimos significativos de produção.

$\mathrm{O} \mathrm{N}$, depois do $\mathrm{C}, \mathrm{H}$ e $\mathrm{O}$, é o elemento químico quantitativamente mais abundante na matéria viva (SIQUEIRA; FRANCO, 1988). As plantas exigem uma grande quantidade deste elemento, porém, grande parte deste elemento está na atmosfera, numa forma de difícil de ser acessada pelas plantas. Algumas bactérias de vida livre ou não conseguem fixar este elemento e transformar $\mathrm{N}_{2}$ para $\mathrm{NH}_{4}$, como, por exemplo, as bactérias do gênero Bradyrhizobium que vivem de uma forma mutualística com a soja.

Em relação à produção de alimentos no mundo, as leguminosas constituem cerca de $9 \%$ em matéria seca, porém representam $24 \%$ do total de proteína (HARDARSON; LIE, 1984).

O objetivo deste trabalho foi avaliar teor de $\mathrm{N}$ foliar, número de nódulos, massa seca de nódulos e de plantas com doses de lodo de esgoto compostado e inoculação da bactéria Bradyrhizobium em plantas de soja.

\section{Material e Métodos}

$\mathrm{O}$ experimento foi conduzido em casa de vegetação do Departamento de Ciência do Solo e Recursos Naturais da Faculdade de Ciências Agronômicas de Botucatu. 
Foi adotado o delineamento experimental em blocos casualizados constituído por cinco doses de lodo de esgoto, com e sem a inoculação do Bradyrhizobium Japonicum, com 3 repetições por tratamento. Cada vaso com 30L de capacidade, contendo 4 plantas de soja, foi considerado uma parcela. T1, T2, T3, T4, T5, receberam as seguintes dosagens de lodo compostado, respectivamente: $0,10,20,30,40 \mathrm{t} \mathrm{ha}^{-1}$ sem inoculação com o bradyrizobium; T6, T7, T8, T9 e T10 receberam as doses de $0,10,20,30,40 \mathrm{t} \mathrm{ha}^{-1}$, respectivamente, com a inoculação do bradyrizobium.
O substrato utilizado nos vasos foi um Latossolo Vermelho Amarelo com textura arenosa (EMBRAPA, 1999), cujas características químicas são apresentadas na Tabela 1.

Foi utilizado o composto de lodo de esgoto proveniente da Estação de Tratamento de Esgoto da cidade de Jundiaí/SP. As análises foram realizadas no Laboratório de Fertilizantes e Corretivos do Departamento de Recursos Naturais / Ciência do Solo da Faculdade de Ciências Agronômica de Botucatu, SP (Tabela 2).

Tabela 1. Características químicas do solo utilizado no experimento.

\begin{tabular}{|c|c|c|c|c|c|c|c|c|c|c|}
\hline \multirow{2}{*}{$\begin{array}{c}\mathrm{pH} \\
\mathrm{CaCl}_{2}\end{array}$} & \multirow{2}{*}{$\begin{array}{l}\text { M.O. } \\
\mathrm{g} \mathrm{dm}^{-3}\end{array}$} & \multirow{2}{*}{$\begin{array}{l}\text { P(res.) } \\
\mathrm{mg} \mathrm{dm}^{-3}\end{array}$} & $\mathrm{H}+\mathrm{Al}$ & $\mathrm{Al}^{+3}$ & $\mathrm{~K}^{+}$ & $\mathrm{Ca}^{+2}$ & $\mathrm{Mg}^{+2}$ & SB & $\mathrm{T}$ & $\mathrm{V}$ \\
\hline & & & \multicolumn{7}{|c|}{ 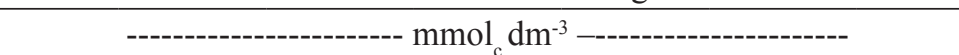 } & $\%$ \\
\hline 4,1 & 18 & 3 & 70 & 13 & 0,5 & 2 & 1 & 3 & 74 & 5 \\
\hline
\end{tabular}

Fonte: $\mathrm{pH}$ em $\mathrm{CaCl}_{2}$, relação 1: 2,5; método de Walkley-Black; $\mathrm{H}+\mathrm{Al}$ - método potenciométrico (solução tampão $\mathrm{SMP}$ ); $\mathrm{Ca}$, $\mathrm{Mg}$, $\mathrm{K}$ - extrator: resina trocadora de íons; $\mathrm{Al}$ - extrator: $\mathrm{KCl} ; \mathrm{SB}=$ Soma de bases; $\mathrm{T}=\mathrm{SB}+\mathrm{H}+\mathrm{Al} ; \mathrm{V}=\mathrm{SB} / \mathrm{T} * 100$.

Tabela 2. Características químicas do lodo compostado.

\begin{tabular}{|c|c|c|c|c|c|c|c|c|c|c|c|c|c|c|c|}
\hline $\mathrm{N}$ & $\mathrm{P}_{2} \mathrm{O}_{5}$ & $\mathrm{~K}_{2} \mathrm{O}$ & Umid. & $\mathrm{MO}$ & C & $\mathrm{Ca}$ & $\mathrm{Mg}$ & S & $\mathrm{Na}$ & $\mathrm{Cu}$ & $\mathrm{Fe}$ & $\mathrm{Mn}$ & $\mathrm{Zn}$ & $\mathrm{C} / \mathrm{N}$ & $\mathrm{pH}$ \\
\hline ---- & & & $---\% \mathrm{r}$ & mat & ia sec & 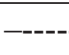 & & 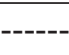 & \multicolumn{5}{|c|}{---- $\mathrm{mg} \mathrm{kg}^{-1}$ na matéria seca ----- } & & \\
\hline 2,44 & 2,0 & 0,4 & 58 & 53 & 29,4 & 1,9 & 0,3 & 1,6 & 1340 & 304 & 23250 & 472 & 3750 & $12 / 1$ & 6,6 \\
\hline
\end{tabular}

Fonte: Concentrações totais de nutrientes determinados no extrato da digestão nítricoperclorica.

O solo foi peneirado e recebeu uma dose de calcário calculada para elevar a V\% a 60, e em seguida foi feita uma irrigação para atingir $70 \%$ da capacidade de campo, sendo o solo assim tratado incubado por 60 dias. Após este período foi adubado com 5,6 g de $\mathrm{P}_{2} \mathrm{O}_{5}$ e 4,2 $\mathrm{g} \mathrm{K}_{2} \mathrm{O}$ na forma de superfosfato simples e cloreto de potássio por vaso, respectivamente, e foram realizadas as incorporações de composto de lodo nas doses estipuladas.

Após uma semana da adubação foi efetuada a semeadura da soja, variedade Conquista, com espaçamento de $5 \mathrm{~cm}$ entre uma semente e outra.
Aos 20 dias da semeadura mantiveram-se 5 plantas por vaso e a partir dos 30 dias apenas 4 plantas por vaso.

Para o controle da Diabrotica brasiliensis (Vaquinha) foram feitas duas aplicações com Deltamethrin na dosagem de $2 \mathrm{~mL}$ em $2 \mathrm{~L}$ de água, aos 20 dias e aos 27 dias da semeadura. Aos 83 dias da semeadura foi efetuado o controle da Anticarsia gemmatalis (lagarta da soja), também com Deltamethrin. Para o controle da Nicrosphaera diffusa (oídio) foi efetuada uma aplicação de Tebucanazole na dosagem de $1 \mathrm{~mL}$ em $4 \mathrm{~L}$ aos 20, 27 e 34 dias após a semeadura. Para o controle da Tetranychus urticae (ácaro rajado) foi utilizado o 
Spiromesifen aos 20 e aos 27 dias após a semeadura, na dosagem de $1 \mathrm{~mL}$ em $4 \mathrm{~L}$ de água.

As irrigações foram efetuadas diariamente para atingir $70 \%$ da capacidade de campo, esta medida foi efetuada com um vazo que estava sem planta somente com o solo e este era pesado diariamente verificando desta forma a diferença de peso do vazo com o ideal ( $70 \%$ da capacidade de campo).

Por ocasião do início da floração (77 dias da semeadura), foram coletadas duas amostras de folhas de soja de cada vaso (sempre escolhendo a primeira folha a partir da ponta do ramo), sendo posteriormente levadas à estufa com circulação de ar forçada, até atingir massa constante. Em seguida as folhas foram moídas e analisadas em Laboratório de Nutrição Mineral de Plantas, do Departamento de Recursos Naturais/ Ciência do Solo/ Botucatu/ SP, determinando os teores de N (MALAVOLTA; VITTI; OLIVEIRA, 1997).

Aos 77 dias foram cortadas as partes aéreas das plantas e colocadas na estufa até atingir massa constante, para determinar a matéria seca. Foram retirados os nódulos das raízes e armazenados em sacos de plástico identificados e colocados em geladeira. Posteriormente, foi contado o número de nódulos e estes foram transferidos para estufa e, depois de secos, pesados.

Os dados coletados foram submetidos à análise de variância e ao teste de média nos tratamentos que receberam a mesma dose de lodo compostado por meio do sistema estatístico SAEG (1993). Foi efetuada analise de regressão para as doses do composto de lodo de esgoto separadamente para os tratamentos com e sem a inoculação com Bradyrhizobium.

\section{Resultados e Discussão}

Verifica-se que os tratamentos que receberam lodo compostado sem inoculação com Bradyrhizobium e os tratamentos que receberam uma dosagem de 30 e $40 \mathrm{t} \mathrm{ha}^{-1}$ de lodo, com as sementes inoculadas, apresentaram valores de concentração de $\mathrm{N}$ abaixo do adequado (Tabelas 3, 4, 5, 6 e 7). Segundo Malavolta, et al. (1999), a concentração adequada de $\mathrm{N}$ na folha, para uma produtividade de $2,7 \mathrm{t} \mathrm{ha}^{-1}$ de soja, seria de $36 \mathrm{~g} \mathrm{~kg}^{-1}$. Verifica-se na Tabela 3 que não houve diferença no teor foliar de $\mathrm{N}$ nas plantas que receberam a inoculação com Bradyrhizobium com a sem inoculação nos tratamentos que não receberam lodo de esgoto compostado. Nas Tabelas 4,5 e 6 onde foi utilizado as dosagens de lodo de 10,20 e $30 \mathrm{t} \mathrm{ha}^{-1}$, respectivamente, verifica-se que as sementes que foram inoculadas apresentaram teores foliares de $\mathrm{N}$ superiores que as sementes que não foram inoculadas. Na Tabela 7 verifica-se que na dose de $40 \mathrm{t} \mathrm{ha}^{-1}$ a soja inoculada apresentou um teor foliar de $\mathrm{N}$ inferior a soja sem inoculação. Nas plantas em que as sementes não foram inoculadas com Bradyrhizobium ocorreu um decréscimo no teor de $\mathrm{N}$ nas folhas com o aumento da dose de lodo até $30 \mathrm{t} \mathrm{ha}^{-1}$ apresentando nesta dose um teor de $19,16 \mathrm{~g} \mathrm{~kg}^{-1}$ e, após esta dose, ocorreu um aumento do teor foliar de N (Figura 1). Dentre as plantas que foram inoculadas com Bradyrhizobium ocorreu um aumento no teor de $\mathrm{N}$ até a dose de $11 \mathrm{tha}^{-1}$ de lodo com um teor de 49,71 $\mathrm{g} \mathrm{kg}^{-1} \mathrm{e}$, acima desta dosagem, houve um decréscimo (Figura 1). Isto pode ser explicado pelo fato de que, quando aumentamos o N no solo, seja este mineral ou orgânico, a fixação biológica começa a decair (MOREIRA; SIQUEIRA, 2006). Correa et al. (2008) observaram que, com o aumento das doses de resíduos orgânicos (lodo de esgoto centrifugado, lodo de biodigestor, escória de aciaria e lama de cal), ocorre aumento do teor de $\mathrm{N}$ nas folhas de soja nas dosagens desses resíduos de $0,2,4,6$ e $8 \mathrm{t} \mathrm{ha}^{-1}$, uma dosagem bem menor do que as utilizadas neste experimento.

Observa-se nas Tabelas 4, 6 e 7 que as plantas provenientes de sementes inoculaladas apresentaram um maior número de nódulos nas doses de lodo de 10,30 e $40 \mathrm{t} \mathrm{ha}^{-1}$ e nas demais doses utilizadas não diferiram. Sem a inoculação das sementes ocorreu um aumento do $\mathrm{n}^{\mathrm{o}}$ de nódulos até a dose de $7 \mathrm{t}$ ha 1, apresentando nesta dose 344 número de nódulos 
nas quatro plantas, e acima desta dose ocorreu um decréscimo no $\mathrm{n}^{\mathrm{o}}$ de nódulos (Figura 2). Nas plantas que as sementes foram inoculadas, observou-se aumento do número de nódulos até a dose de $19 \mathrm{t}$ ha $^{-1}$ de lodo de esgoto com 569 numero de nódulos nas quatro plantas e acima desta dose ocorreu uma diminuição do $\mathrm{n}^{\mathrm{o}}$ de nódulos (Figura 2). Hungria, Campo e Mendes (2003) não observaram diferenças significativas no número de nódulos de feijoeiros inoculados com rizóbios eficientes e de plantas supridas com $\mathrm{N}$ mineral (30 $\mathrm{kg} \mathrm{ha}^{-1}$ na semeadura $+30 \mathrm{~kg} \mathrm{ha}^{-1}$ em cobertura), noduladas apenas com as populações nativas do solo na região sul do país. Entretanto, Peres et al. (1994) obtiveram incrementos significativos na nodulação de feijoeiros em três ensaios com a inoculação de estirpes comerciais de rizóbios na cultivar Carioca e diminuição da nodulação com a aplicação de N mineral.

Tabela 3. Teor de $\mathrm{N}$ nas folhas, $\mathrm{N}^{\mathrm{o}}$ de nódulos, massa seca dos nódulos e massa seca no florescimento em quatro na dose de 0 t ha-1 com e sem inoculação.

\begin{tabular}{|c|c|c|c|c|}
\hline Tratamentos & $\begin{array}{l}\text { Teor de N nas Folhas } \\
\left(\mathrm{g} \mathrm{kg}^{-1}\right)\end{array}$ & $\begin{array}{l}\mathrm{N}^{\mathrm{o}} \text { de nódulos } \\
\text { unidade }\end{array}$ & $\begin{array}{ll}\text { Peso seco dos } \\
\text { nódulos gramas }\end{array}$ & $\begin{array}{l}\text { Matéria seca parte } \\
\text { aérea gramas }\end{array}$ \\
\hline Sem inoculante & 37,00 & 386,33 & 3,38 & 51,34 \\
\hline Com inoculante & 39,33 & 322,67 & 3,42 & 55,85 \\
\hline $\mathrm{F}$ & 2,23 & 0,652 & 0,003 & 0,62 \\
\hline Média & 38,17 & 354,50 & 3,40 & 53,59 \\
\hline $\mathrm{CV}$ & 5,02 & 27,24 & 25,58 & 13,02 \\
\hline
\end{tabular}

Fonte: Elaboração dos autores.

Tabela 4. Teor de $\mathrm{N}$ nas folhas, $\mathrm{N}^{\mathrm{o}}$ de nódulos, massa seca dos nódulos e massa seca no florescimento em quatro na dose de $10 \mathrm{t} \mathrm{ha}^{-1}$ com e sem inoculação.

\begin{tabular}{llllll}
\hline Tratamentos & $\begin{array}{l}\text { Teor de N nas Folhas } \\
\left(\mathrm{g} \mathrm{kg}^{-1}\right)\end{array}$ & $\begin{array}{l}\mathrm{N}^{\circ} \text { de } \\
\text { unidade }\end{array}$ & nódulos & $\begin{array}{l}\text { Peso seco dos } \\
\text { nódulos gramas }\end{array}$ & $\begin{array}{l}\text { Matéria seca parte } \\
\text { aérea gramas }\end{array}$ \\
\hline Sem inoculante & $24,33 \mathrm{~b}$ & $176 \mathrm{~b}$ & 3,21 & 50,52 \\
Com inoculante & $42,33 \mathrm{a}$ & $527,33 \mathrm{a}$ & 4,18 & 59,19 \\
\hline F & 10,52 & 55,55 & 7,38 & 7,38 \\
Média & 31,67 & 351,67 & 3,70 & 54,85 \\
CV & 17,49 & 16,42 & 11,79 & 7,12 \\
\hline
\end{tabular}

Fonte: Elaboração dos autores.

Tabela 5. Teor de $\mathrm{N}$ nas folhas, $\mathrm{N}^{\mathrm{o}}$ de nódulos, massa seca dos nódulos e massa seca no florescimento em quatro na dose de $20 \mathrm{t} \mathrm{ha}^{-1}$ com e sem inoculação.

\begin{tabular}{llllll}
\hline Tratamentos & $\begin{array}{l}\text { Teor de N nas Folhas } \\
\left(\mathrm{g} \mathrm{kg}^{-1}\right)\end{array}$ & $\begin{array}{l}\mathrm{N}^{\circ} \text { de } \\
\text { unidade }\end{array}$ & nódulos & $\begin{array}{l}\text { Peso seco dos } \\
\text { nódulos gramas }\end{array}$ & $\begin{array}{l}\text { Matéria seca parte } \\
\text { aérea gramas }\end{array}$ \\
\hline Sem inoculante & $23,33 \mathrm{~b}$ & 455,33 & 4,34 & 56,33 \\
Com inoculante & $39,00 \mathrm{a}$ & 565,33 & 4,87 & 70,33 \\
\hline F & 22,09 & 6,41 & 2,18 & 3,40 \\
Média & 31,17 & 510,33 & 4,60 & 63,33 \\
CV & 13,10 & 10,42 & 9,67 & 14,68 \\
\hline
\end{tabular}

Fonte: Elaboração dos autores. 
Figura 1. Teor foliar de $\mathrm{N}$ na soja sem inoculação e com inoculação do bradyrhizobium em respostas a doses de lodo de esgoto compostado.

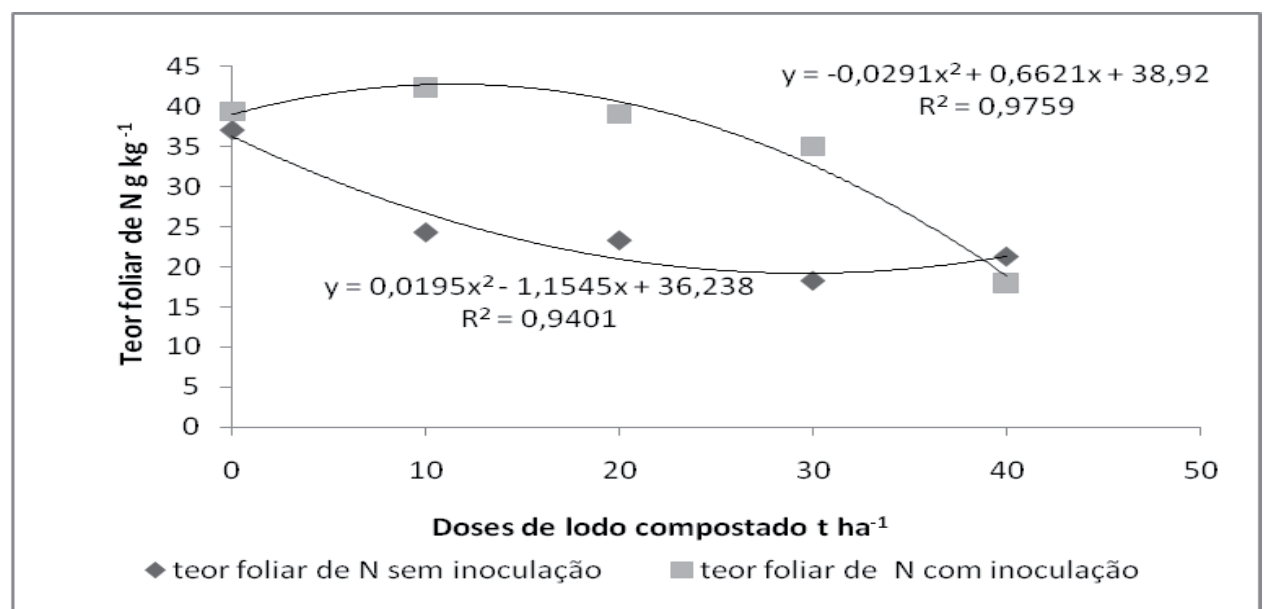

Fonte: Elaboração dos autores.

Figura 2. Número de nódulos de bradyrhizobium na soja sem inoculação e com inoculação do bradyrhizobium em respostas a doses de lodo de esgoto compostado.

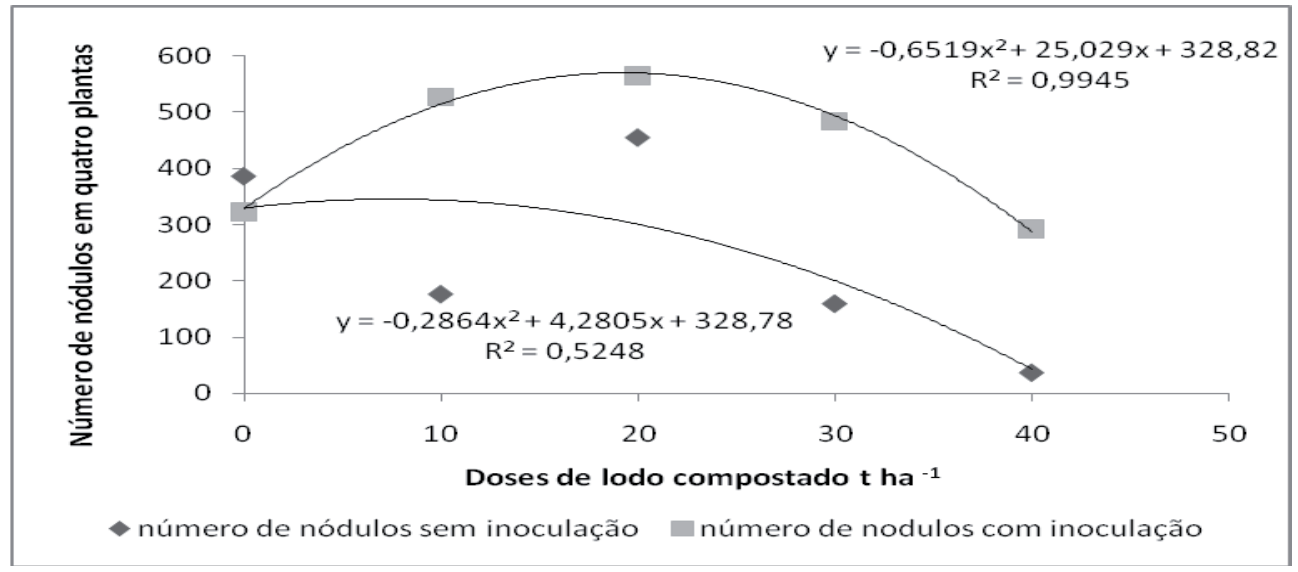

Fonte: Elaboração dos autores.

Na dose de 30 e 40 t ha $^{-1}$, observa-se na Tabela 6 e 7 que as plantas provenientes de sementes que foram inoculadas apresentaram uma maior massa seca dos nódulos. Nas plantas sem inoculação de rizóbio a massa seca dos nódulos aumentou até a dosagem de $12 \mathrm{tha}^{-1}$ com o peso de massa seca nas quatro plantas de 3,89 $\mathrm{g}$ e depois deste valor ocorreu um decréscimo (Figura 3). Já as plantas inoculadas com o rizóbio apresentaram um aumento da massa seca dos nódulos até a dosagem de $17 \mathrm{t} \mathrm{ha}^{-1}$ de lodo compostado com uma massa seca nesta dose de 5,07 g, enquanto acima desta dosagem ocorreu um decréscimo (Figura 3). O lodo de esgoto pode conter numerosos nutrientes para o crescimento microbiano, o que pode aumentar a atividade de microrganismos do solo, inclusive a dos rizóbios (ABD-ALLA; YAN; SCHUBERT, 1999). O número de nódulos e a massa seca dos nódulos são indicadores usuais de nodulação (FERREIRA; CASTRO, 1995) e a presença de nódulos menores e em pequena quantidade pode ser um indicativo de condições ambientais adversas (VARGAS et al., 
2004). Vieira, Tanaka e Silva (2004) constataram que a aplicação de lodo de esgoto estimulou a nodulação, assim como a FBN, estimada por meio da avaliação da atividade da nitrogenase em ensaios de casa de vegetação. Currie, Angle e Hill (2003) também demonstraram em experimento realizado em campo, que a aplicação de lodo de esgoto pode incrementar as taxas de FBN na cultura da soja. Souza et al. (2009) concluíram que uma aplicação do lodo de esgoto em doses de até $6 \mathrm{t} \mathrm{ha}^{-1}$ não afeta negativamente a nodulação da soja no período de dois anos.

Tabela 6. Teor de $\mathrm{N}$ nas folhas, $\mathrm{N}^{\mathrm{o}}$ de nódulos, massa seca dos nódulos e massa seca no florescimento em quatro na dose de $30 \mathrm{t} \mathrm{ha}^{-1}$ com e sem inoculação.

\begin{tabular}{llllll}
\hline Tratamentos & $\begin{array}{l}\text { Teor de N nas Folhas } \\
\left(\mathrm{g} \mathrm{kg}^{-1}\right)\end{array}$ & $\begin{array}{l}\mathrm{N}^{\mathrm{o}} \text { de } \\
\text { unidade }\end{array}$ & $\begin{array}{l}\text { nódulos } \\
\text { neso seco dos gramas }\end{array}$ & $\begin{array}{l}\text { Matéria seca parte } \\
\text { aérea gramas }\end{array}$ \\
\hline Sem inoculante & $18,33 \mathrm{~b}$ & $159 \mathrm{~b}$ & $1,96 \mathrm{~b}$ & $45,38 \mathrm{~b}$ \\
Com inoculante & $35,00 \mathrm{a}$ & $484 \mathrm{a}$ & $4,87 \mathrm{a}$ & $63,31 \mathrm{a}$ \\
\hline F & 73,53 & 15,68 & 186,74 & 50,67 \\
Média & 26,67 & 321,50 & 3,42 & 54,34 \\
CV & 8,93 & 31,26 & 7,62 & 5,67 \\
\hline
\end{tabular}

Fonte: Elaboração dos autores.

Tabela 7. Teor de $\mathrm{N}$ nas folhas, $\mathrm{N}^{\mathrm{o}}$ de nódulos, massa seca dos nódulos e massa seca no florescimento em quatro na dose de $40 \mathrm{tha}^{-1}$ com e sem inoculação.

\begin{tabular}{llllll}
\hline Tratamentos & $\begin{array}{l}\text { Teor de N nas Folhas } \\
\left(\mathrm{g} \mathrm{kg}^{-1}\right)\end{array}$ & $\begin{array}{l}\mathrm{N}^{\mathrm{o}} \text { de } \\
\text { unidade }\end{array}$ & $\begin{array}{l}\text { nódulos } \\
\text { Sem inoculante }\end{array}$ & $\begin{array}{l}\text { Peso seco } \\
\text { nódulos gramas }\end{array}$ & $\begin{array}{l}\text { dos } \\
\text { atéria seca parte } \\
\text { areamas }\end{array}$ \\
\hline F & $36 \mathrm{~b}$ & $0,07 \mathrm{~b}$ & 62,31 \\
Média & $25,00 \mathrm{~b}$ & $292 \mathrm{a}$ & $1,51 \mathrm{a}$ & 63,75 \\
CV & 19,00 & 223,93 & 992,69 & 0,046 \\
\hline
\end{tabular}

Fonte: Elaboração dos autores.

Figura 3. Massa seca dos nódulos na soja sem inoculação e com inoculação do bradyrhizobium em respostas a doses de lodo de esgoto compostado.

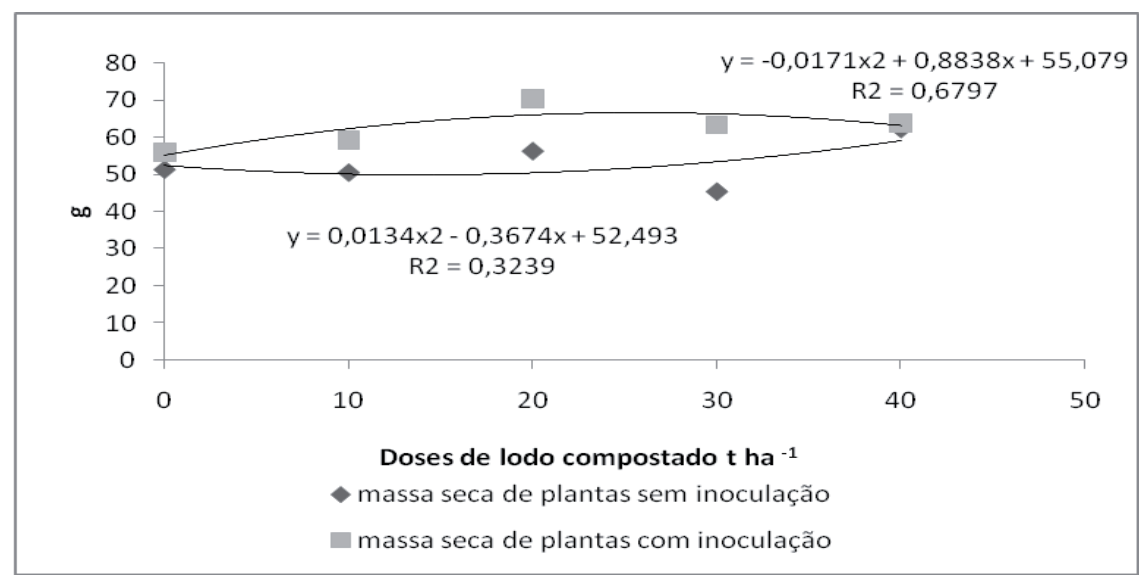

Fonte: Elaboração dos autores. 
Vieira et al. (1995) relatam os efeitos positivos do Mo em leguminosas por aumentar a atividade específica da nitrogenase e ser cofator da nitratoredutase. É possível que o lodo de esgoto compostado tenha aumentado a disponibilidade deste nutriente importante para a fixação biológica do $\mathrm{N}$ na soja, embora a quantidade necessária deste nutriente seja muito pequena, sendo que as sementes com alto teor de Mo dispõem de quantidades suficientes para suprir a exigência das plantas (MEAGHER; JOHNSON; STOUT, 1952). Entretanto, esta é apenas uma hipótese com relação ao efeito do lodo de composto utilizado, visto que não há dados disponíveis sobre este assunto.

Teixeira et al. (2006) relataram que a adição de lodo de curtume em doses elevadas em feijão caupi apresentou um efeito negativo sobre a nodulação da bactéria inoculada, enquanto Pelegrini et al. (2009) constataram que, com o aumento da dose de $\mathrm{N}$ em feijoeiro comum, ocorreu uma diminuição da nodulação (número e massa seca dos nódulos); isto demonstra que o excesso de $\mathrm{N}$ pode inibir a nodulação e acredita-se que devido a isto o tratamento que recebeu a maior dose de lodo compostado apresentou um menor número e massa de nódulos.

Em trabalho realizado com soja, em casa de vegetação, a aplicação equivalente a $23 \mathrm{t} \mathrm{ha}^{-1}$ de lodo de esgoto ao solo (base úmida) prejudicou a fixação biológica pelo alto teor de N (VIEIRA, 2001). Nesse trabalho, como o lodo de esgoto foi aplicado em plantas inoculadas e sem inoculação, ficou evidente a importância do processo simbiótico na resposta das plantas ao lodo de esgoto, ou seja, as maiores produtividades foram obtidas pelas plantas que receberam lodo de esgoto, mas que também foram submetidas à inoculação

A produtividade de massa seca da parte aérea na dose de 30 t ha $^{-1}$ de lodo compostado apresentouse superior nas plantas provindas de sementes inoculadas do que nas sementes que não receberam a inoculação (Tabela 6).

O aumento da dose de lodo compostado em plantas que não foram inoculadas com o rizóbio resultou em aumento no rendimento de massa seca (Figura 4). O rendimento de matéria seca de plantas inoculadas aumentou até a dosagem de $26 \mathrm{t} \mathrm{ha}^{-1}$ de lodo compostado com 66,50 gramas de massa seca em 4 plantas e, após esta dosagem, ocorreu um decréscimo no rendimento. Sabe-se que há correlação positiva entre a produção de matéria seca no estádio de florescimento com a produção final de grãos e, portanto, é possível assumir que as plantas mais produtivas em um desses atributos, também o serão no outro.

Figura 4. Rendimento de massa seca na soja sem inoculação e com inoculação do bradyrhizobium em respostas a doses de lodo de esgoto compostado.

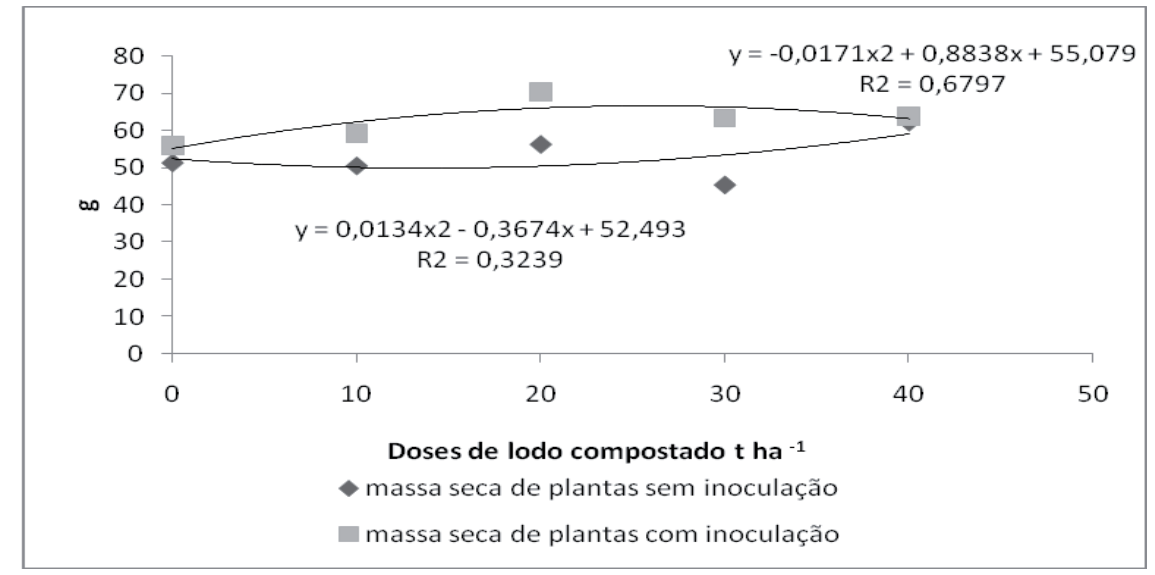

Fonte Elaboração dos autores. 
Em média o aproveitamento do lodo de esgoto como fertilizante na cultura da soja é viável em termos agronômicos e econômicos de acordo com Lemainski e Silva (2006). O lodo de esgoto, em média, é $18 \%$ mais eficiente do que o fertilizante mineral como fonte de nutrientes na cultura da soja.

A prática de aliar a incorporação de lodo de esgoto compostado nessas doses, à inoculação das sementes com rizóbio é uma prática que deve ser recomendada para a obtenção da maior produtividade em soja e a melhor fixação biológica do N.

\section{Conclusões}

A inoculação com Bradyrhizobium obteve resultados superiores que a não inoculação na dose acima de 10 t ha $^{-1}$ de lodo de esgoto compostado.

A melhor dose de lodo de esgoto compostado nas sementes inoculadas com Bradyrhizobium para o teor de $\mathrm{N}$ foliar, número de nódulos na raiz, massa seca de nódulos e massa da matéria seca foram: 11, 19, 17 e $26 \mathrm{t} \mathrm{ha}^{-1}$, respectivamente.

Para as sementes que não receberam a inoculação com Bradyrhizobium o aumento da dose de lodo de esgoto compostado obteve um decréscimo nos parâmetros avaliados.

\section{Referências}

ABD-ALLA, H. M.; YAN, F.; SCHUBERT, S. Effect of sewage sludge application on nodulation, nitrogen fixation, and plant growth of faba bean, soybean and lupin. Journal of Applied Botany, Gottingen, v. 73, n. 1, p. $69-75,1999$.

BERTON, R. S.; NOGUEIRA, T. A. R. Uso de lodo de esgoto na agricultura. In: COSCIONE, A. R.; NOGUEIRA, T. A. R.; PIRES, A. M. M. Uso agrícola de lodo de esgoto: avaliação após a resolução $n^{\circ} 375$ do CONAMA. Botucatu: FEPAF, 2010. cap. 2, p. 31-50.

BETTIOL, W.; CAMARGO, O. A. de Impacto ambiental do uso agrícola do lodo de esgoto. In: SPADOTTO, C.; RIBEIRO, W. Gestão de resíduos na agricultura e agroindustria. Botucatu: FEPAF, 2006. cap. 8, p. 181204.

BOEIRA, R. C. Uso do lodo de esgoto como fertilizante orgânico: disponibilização de nitrogênio em solos tropical. Jaguariúna: Embrapa Meio Ambiente, 2004. (Comunicado técnico, 12).

CAMPO, R. J.; PALHANO, J. B.; LANTMANN, A. F. Influencia da aplicação de dose de nitrogênio sobre o processo de fixação simbiótica do nitrogênio. In: EMBRAPA. Centro nacional de pesquisa de soja. Londrina, PR. Resultados de pesquisa de soja 1980/81. Londrina: Embrapa, 1981. p. 218-221.

CAMPO, R. J.; SFREDO, G. J. Nitrogênio na cultura da soja. Londrina: EMBRAPA-CNPSo, 1981, 6 p. (EMBRAPA-CNPSo. Comunicado técnico, 8).

CETESB Aplicação de lodo de sistema de tratamento em áreas agrícolas; critério para projeto e operação. São Paulo: CETESB NORMA, 1999. 32 p. (Manual técnico).

CORDEIRO, D. S. Efeito de adubação NPK na absorção, translocação e extração de nutrientes pela soja (Glycine Max (L.) Merrill). 1977. Tese (Doutorado em Solos e Nutrição de Plantas) - Escola Superior de Agricultura Luiz de Queiroz, Piracicaba.

CORREA, J. C.; BULL, L. T.; CRUSCIOL, C. A. C.; TECCHIO, M. A. Aplicação superficial de escoria, lama cal, lodos de esgoto e calcário na cultura da soja. Pesquisa Agropecuária Brasileira, Brasília, v. 43, n. 9, p. 1209-1219, 2008.

CURRIE, V. C.; ANGLE, J. S.; HILL, R. L. Biosolids application to soybeans and effects on input and output of nitrogen. Agriculture, Ecosystems and Environment, Amsterdan, v. 97, n. 1, p. 345-351, 2003.

EMPRESA BRASILEIRA DE PESQUISA AGROPECUARIA - EMBRAPA. Produtores rurais conhecem uso do lodo de esgoto na agricultura. Brasília: EMBRAPA/CERRADOS, 2007. 2 p.

. Sistema brasileiro de classificação de solos. Rio de Janeiro: EMBRAPA/CNPSO, 1999. 412 p.

FERREIRA, E. M.; CASTRO, I. V. nodulation and growth of subterranean clover (Trifolium subterraneum L.) in soils previously treated with sewage sludge. Soil Biology and Biochemistry, Oxford, v. 27, n. 1, p. 11771183, 1995.

HARDARSON, G.; LIE, T. A. (Ed.). Breeding legumes for enhanced symbiotic nitrogen fixation. Proceeding of a FAO/IEAA Consultants Meeting, Vienna, 1983. Plant and Soil, The Hague, v. 82, n. 3, p. 273-448, 1984. 
HUNGRIA, M.; CAMPO, R. J.; MENDES, I. Benefits of inoculation of the common bean (Phaseolus vulgaris) crop with efficient and competitive Rhizobium tropici strains. Biology Fertility Soils, Berlin, v. 39, n. 2, p. 8893, 2003.

LEMAINSKI, J.; SILVA, J. E. da. Avaliação agronômica e econômica da aplicação de biossólido na produção de soja. Pesquisa Agropecuária Brasileira, Brasília, v. 41. n. 10, p. 1477-1484, 2006.

MALAVOLTA, E.; VITTI, G. C.; OLIVEIRA, S. A. Avaliação do estado nutricional das plantas: princípios, métodos e técnicas de avaliação do estado nutricional. 2. ed. Piracicaba: Potafós, 1997. 319 p.

MALAVOLTA, E.; OLIVEIRA, S. A.; WADT, P. G. S. Foliar diagnosis: the status of the art. In: SIQUEIRA, J. O.; MOREIRA, F. M. S.; LOPES, A. S.; GUILHERME, L. R. G.; FAQUIN, V.; FURTINI NETO, A. E.; CARVALHO, J. G. (Ed.). Inter-relação fertilidade, biologia do solo e nutrição de plantas. Viçosa: SBCS, 1999. p. 205-242.

MEAGHER, W. R.; JOHNSON, C. M.; STOUT, P. R. Molybdenum requirements of leguminous plants supplied with fixed nitrogen. Plant Physiology, Illinois, v. 27, n. 2, p. 223-230, 1952.

MOREIRA, F. M. S.; SIQUEIRA, J. O. Fixação biológica de nitrogênio atmosférico. In: MOREIRA, F. M. S.; SIQUEIRA, J. O. Microbiologia e bioquímica do solo. Lavras: UFLA, 2006. cap. 9, p. 449-542.

PELEGRIN, R. de; MERCANTE, F. M.; OTSUDO, I. M. N.; OTSUDO, A. A. Resposta da cultura do feijoeiro á adubação nitrogenada e à inoculação com rizóbio. Revista Brasileira de Ciência do Solo, Viçosa, MG, v. 33, n. 1, p. 219-226, 2009.

PERES, J. R. R.; SUHET, A. R.; MENDES, I. C.; VARGAS, M. A. T. Efeito da inoculação com rizóbio e da adubação nitrogenada em sete cultivares de feijão em solos de cerrado. Revista Brasileira de Ciência do Solo, Viçosa, MG, v. 18, n. 3, p. 415-420, 1994.

SIQUEIRA, J. O.; FRANCO, A. A. Fixação biológica de nitrogênio. In: SIQUEIRA, J. O. Biotecnologia do solo fundamentos e perspectivas. Brasília: Gráfica Nagy Ltda., 1988. cap. 6, p. 179-216.
UNIVERSIDADE FEDERAL DE VIÇOSA - UFV. SAEG - Sistema de análises estatísticas e genéticas. Viçosa, MG: Fundação Arthur Bernardes, 1993.

SOUZA, C. A. de; REIS JUNIOR, F. B. dos; MENDES I de C.; LEMAINSKI, J.; SILVA, J. E. da. Lodo de esgoto em atributos biológicos do solo e na nodulação e produção de soja. Pesquisa Agropecuária Brasileira, Brasília, v. 44, n. 10, p. 1319-1327, 2009.

TEIXEIRA, K. R. G.; GONÇALVES FILHO, L. A. R.; CARVALHO, E. M. S.; ARAUJO, A. S. F. de; SANTOS, V. B. dos. Efeito da adição de lodo de curtume na fertilidade do solo, nodulação e rendimento de matéria seca do caupi. Ciência Agrotecnologia, Lavras, v. 30, n. 6, p. 1071-1076, 2006.

VARGAS, M. A. T.; MENDES, I. C.; CARVALHO, A. M.; LOBO-BURLE, M.; HUNGRIA,M. Inoculação de leguminosas e manejo de adubos verdes. In: SOUSA, D. M. G.; LOBATO, E. Cerrado: correção do solo e adubação. Brasília, DF: Embrapa, 2004. p. 97-128.

VIEIRA, R. F. Sewage sludge effect on soyben growth and nitrogen fixation. Biology and Fertility of Soils, Berlin, v. 34, n. 3, p. 196-200, 2001.

VIEIRA, R. F.; CARDOSO, E. J. B. N.; VIEIRA, F.; CASSINI, S. T. A. Foliar application of molybdenum in common beans. I. Nitrogenase and nitrate reductase activies in a soil of high fertility. In: INTERNATION SYMPOSIUM ON SUBSTAINABLE AGRICULTURE FOR THE TRAPHICS - THE ROLE OF BIOLOGICAL NITROGEN FIXATION, 1., 1995, Rio de Janeiro. Anais... Rio de Janeiro: Embrapa-CNPAB, 1995. p. 189190.

VIEIRA, R. F.; TANAKA, R. T.; SILVA, C. M. M. S. Utilização do lodo de esgoto na cultura da soja. Jaguariúna: Embrapa meio Ambiente. 2004. 26 p. (Embrapa meio ambiente. Boletim de Pesquisa e Desenvolvimento, 21). 